\title{
Hyponatremia as Parameter in Admission and Discharge Disability of Stroke Survivors, Babol, North of Iran
}

\author{
Saadat $\mathrm{P}^{1}$, Ahangar $\mathrm{AA}^{* 1}$, Haghshenas $\mathrm{T}^{2}$, Alijanpour $\mathrm{S}^{3,4}$ and Abolfazl Rahmani ${ }^{5}$ \\ ${ }^{1}$ Mobility Impairment Research Center, Health Research Institute, Babol University of Medical Sciences, Babol, Iran \\ ${ }^{2}$ General Physician, Ayatollah Rouhani Hospital, Babol University of Medical Science, Babol, Iran \\ ${ }^{3} \mathrm{MSc}$ student of critical care nursing, student research committee, Faculty of Nursing and Midwifery, Isfahan univer- \\ sity of medical science, Isfahan, Iran \\ ${ }^{4}$ Education, Research and Planning Unite, Pre-hospital Emergency Organization and Emergency Medical Service \\ Center, Babol University of Medical Science, Babol, Iran \\ ${ }^{5}$ Medical Students, Student Research Committee, Babol University of Medical Science, Babol, Iran
}

${ }^{*}$ Corresponding author: Ahangar AA, Associated professor of Neurology, Mobility Impairment Research Center, Health Research Institute, Babol University of Medical Sciences, Babol, Iran, Tel: 09111111202, E-mail: ahmadiahangaralijan@yahoo.com

Citation: Saadat P, Ahangar AA, Haghshenas T, Alijanpour S, Abolfazl Rahmani (2019) Hyponatremia as Parameter in Admission and Discharge Disability of Stroke Survivors, Babol, North of Iran. J Neurol Neurol Disord 5(1): 101. doi: 10.15744/2454-4981.5.101

Received Date: February 05, 2019 Accepted Date: December 17, 2019 Published Date: December 19, 2019

\begin{abstract}
Background: Stroke is one of the most common neurological diseases, research on risk factors except classical ones has been going on and hyponatremia is one of these.

Methods: This cross-sectional descriptive analytical study was conducted on consecutive stroke patients to evaluate the association of hyponatremia with of stroke. Different intensities were determined on Stroke Scale NIH (National Institute of Health) (mild 4-1) and (average moderate 15-5) and (moderate to severe 20-16) and (extreme severe 42-21). Determination intensity of stroke after admission and discharge with the Modified Rankin Scale and Barthel index. $0.5 \mathrm{~mL}$ of venous blood were taken to determining the serum concentrations of sodium. The normal ranges were defined $135-145 \mathrm{meq} / \mathrm{L}$.
\end{abstract}

Findings: Of the 125 stroke survivors, 55 patients (45.8\%) were male and 108 cases (86.4 \%) were ischemic stroke. 82 cases (65.5\%) of all patients had hyponatremia. The difference in type of stroke had statistically significant with hyponatremia (14 cases (17\%) hemorrhagic, 68cases (83\%) Ischemic stroke, $\mathrm{p}=0.01$ ). Also, Difference in sex had statistically significant with hyponatremia (49cases (59\%) female, 33cases $(41 \%)$ male, $\mathrm{p}=0.02)$. In hyponatremic patients, the severity score in admission $(\mathrm{p}=0 / 01)$ and disability at discharge time ( $\mathrm{p}=0.02)$ was increased in compare to other groups. Furthermore, there was a statistically significant difference between cigarette smoking and hyponatremia in patients with stroke $(\mathrm{p}=0.03)$.

Conclusion: Prevalence of hyponatremia was high in acute stroke patients and can be considering as a predicting factor of stroke severity at admission and discharge time, with an increase in the degree of disability. It suggests a similar cohort study conduct in multi-centers with larger sample size and long term follow-up.

Keywords: Stroke; Hyponatremia; Severity; Disability

\section{Introduction}

Stroke is one of the leading causes of death worldwide [1]. Among the most debilitating neurological diseases, Stroke is the leading cause of serious long-term disability [2,3]. Two-third of all strokes occurs in the developing countries; in spite of their preventable nature, they are increasingly becoming a major health problem [4]. Based on studies conducted in Iran, the incidence of stroke is about 43-50 patients per 100,000populations [4,5]. In addition to high mortality rate, stroke is one of the most important healthcare issues in the world, due to serious long-term disability which cause many financial burdens it imposes on families and countries [6]. 
According to this content, research on every factor which may be associated with stroke is in process. Some trace elements are necessary to be considered in association with stroke. Hyponatremia is a common electrolyte disorder which is seen in hospitalized patients, associated with increased morbidity and mortality rates [7], but its prevalence is scattered in different populations, according to different types of disease and health care programs in these communities. In addition, there are disagreements about the role of hyponatremia in various diseases [8]. Some previous studies have disclosed that hyponatremia is related to high mortality of stroke in the hospital after 3 and 12 months [9].

Also in some studies report, hyponatremia is an independent predictor of 3 year mortality in first-ever ischemic stroke. Given these issues, the amount of serum levels of sodium can be considered among factors determining the prognosis of ischemic stroke [10]. Despite this evidence, there is still no consensus about how the mechanism of hyponatremia affects the prognosis of various diseases in general and stroke in particular [11]. Also, there is disagreement on the prevalence hyponatremia and the impact rate of hyponatremia on severity and prognosis of stroke.

With regard to the issue, we designed and implemented this study to evaluation relationship of hyponatremia with stroke along with other related variables to take appropriate preventive and therapeutic modalities could be taken which may finally lead to the decrease in mortality and decrease the cost related to disabled survived patients and increase in community health.

\section{Material and Methods}

This cross-sectional analytic study on consecutive stroke patients was conducted at Ayatollah Rouhani Hospital in Babol, North of Iran, Ayatollah Rouhani Hospital is the main medical center for stroke management in Babol city and its surrounding areas. The study was approved by the ethics committee of Babol University of medical science (BUMS). The informed consent was obtained from each participant or the next of kin prior to any interview or neurologic examination. The sample included consecutive stroke patients who were hospitalized during the 6-month period (2016).

Diagnosis of stroke was based on standards of the American Heart Association-American Stroke Association [12]. The stroke in this study was divided into ischemic and hemorrhagic types; the Ischemic Stroke (IS) included thrombotic and embolic (cardiac origin and artery to artery). In addition, according to the ischemic mechanism, the stroke was divided into five groups as large vessel occlusion, small vessel occlusion, cardiac emboli, the stroke of other determined etiology, and the stroke of undetermined etiology. And the hemorrhagic stroke (HS) was divided into Intra Cerebral Hemorrhage (ICH) and Subarachnoid Hemorrhage (SAH) subtypes.

Stroke was diagnosed by a neurologist, based on the patient's history, neurological examination and neuroimaging studies which were performed for all consecutive stroke patients. The severity of stroke was determined on the basis of NIH Stroke Scale (NIHSS) criteria (i.e., scores $\leq 8$ mild stroke; scores 9 - 15 moderate stroke; and scores $\geq 16$ severe stroke [13].

The degree of disability of patients was determined according to the mRS. Criteria as, mild, moderate, and severe [14]. In this study, mild disability included patients who in Grade 2 or less of that, cases with Grade 3 were regarded as moderate disability and Grade 4 as severely impaired. All admitted Stroke patients who were higher than 18 years. Before the end of 24 hours after the onset of stroke, entered in this study. transient ischemic attack (TIA) cases were excluded from the study.

Exclusion criteria for stroke cases were hemiparesis or any focal neurological findings resulting from head trauma, metabolic encephalopathy, brain mass lesions such as brain tumor or brain abscess, hemiplegic migraine attack, and postictal of seizures. In addition, patients with renal failure, liver failure and patients under treatment of corticosteroid drug were excluded. Also, Patients who had pneumonia or used antihypertensive drugs, diuretics as possible causes of hyponatremia, were excluded in the study.

The Blood sample was obtained during the first 24 hours after onset of stroke. $0.5 \mathrm{~mL}$ of venous blood was taken and sodium serum level assessment was performed with Latex particle agglutination test with Hitachi 912 auto analyzer in Ayatollah Rouhani Hospital Laboratory. The serum sodium concentration $\left(\left[\mathrm{Na}^{+}\right]\right)<135 \mathrm{mmol} / \mathrm{L}(135 \mathrm{meq} / \mathrm{L})$ considered as Hyponatremia and 135 to $145 \mathrm{mmol} / \mathrm{L}$ was defined as normal serum levels [15].

Prospective data was collected using a checklist. It included age, gender, onset time of stroke, and admission time in the hospital. Type of stroke, subtypes, and the severity score of stroke in admission time and degree of disability of stroke in admission and at discharge time (3-7day after admission) were recorded through the checklist.

The risk factor histories in stroke survivors such as Hypertension (HTN), Ischemic Heart Disease (IHD), Diabetes Mellitus (DM), Hyperlipidemia (HLP), and smoking were recorded. The blood pressure less than $140 \mathrm{mmHg}$ systolic and diastolic less than 85 mmHg were considered as a normal.

Fasting blood sugar greater than $126 \mathrm{mg} / \mathrm{dl}$ was considered as DM. Triglycerides higher than $150 \mathrm{mg} / \mathrm{dl}$ was considered as high triglycerides, HDL less than $40 \mathrm{mg} / \mathrm{dl}$ in men and $50 \mathrm{mg} / \mathrm{dl}$ in women were considered as lower HDL and LDL higher than 130 $\mathrm{mg} / \mathrm{dl}$ was considered as a high LDL [13]. 
History of ischemic heart disease was obtained from background information history of cardiac medications, and ECG. Smoking (cigarettes) was defined as a daily intake of at least 5 cigarettes for at least a year earlier. Patients with at least one major risk factor were considered as patients with positive risk factor. Regarding the fact that smoking as a risk factor in the analysis of the findings was significantly associated with hyponatremia, it was considered as a separate risk factor. Stroke patients were divided into two groups: normal serum level of sodium and hyponatremia.

The 125 stroke patients during a 6 month (2016) period were studied. Considering the incidence of stroke in Babol Northern of Iran [5], which are 50 per hundred thousand populations. This sample sizes are suitable for this period of time.

The collected data were then entered into (SPSS, Inc, Chicago, Illinois, USA, software version 21) and analyzed by t-test for continuous variables and Chi-square tests for categorical variables. P-value less than 0.05 were considered statistically significant.

\section{Finding}

From 138 consecutive stroke patients during a 6-month period in 2016, after exclusion of cases that did not meet the criteria for entering the study125 cases were studied. 70 cases (56\%) were female, and 55 cases (44\%) were male. The difference in sex with Hyponatremia was statistically significant (female 49(39.2) vs. male 33(26.4), $\mathrm{p}=0.02$ ).

108 patients $(86.4 \%)$ ischemic, 85 patients $(68 \%)$ thrombotic and23 patients (18.4\%) were embolic. According to TOAST classification, 42 ischemic cases (49\%) large vessel occlusion, 28 cases (26\%) small vessel occlusion, 11 cases (10\%) cardiac emboli and 27 ischemic cases (15\%) were other determined or undetermined etiology (Table 1).

\begin{tabular}{|c|c|c|c|c|c|}
\hline Variable & Category & $\begin{array}{l}\text { Normal So- } \\
\text { dium }^{\star} \text { N(\%) }\end{array}$ & $\begin{array}{c}\text { Hyponatremia } \\
\mathrm{N}(\%)\end{array}$ & $\begin{array}{l}\text { Total } \\
\mathrm{N}(\%)\end{array}$ & \\
\hline \multirow{2}{*}{$\begin{array}{l}\text { Gender } \\
125(100)\end{array}$} & female & $21(30)$ & $49(70)$ & $70(56)$ & \multirow[t]{2}{*}{0.24} \\
\hline & Male & $22(40)$ & $33(60)$ & $55(44)$ & \\
\hline \multirow{2}{*}{$\begin{array}{l}\text { Ischemic } \\
108(86.4)\end{array}$} & thrombotic & $31(36)$ & $54(64)$ & $85(68)$ & \multirow[t]{2}{*}{0.81} \\
\hline & Embolic & $9(40)$ & $14(60)$ & $23(18.4)$ & \\
\hline \multirow[t]{4}{*}{ TOAST Definition } & Large vessel occlusion & $16(38)$ & $26(62)$ & $42(49)$ & \multirow[t]{4}{*}{0.76} \\
\hline & Small vessel occlusion & $10(36)$ & $18(64)$ & $28(26)$ & \\
\hline & cardiac emboli & $4(36)$ & $7(64)$ & $11(10)$ & \\
\hline & Other and undetermined & $10(37)$ & $17(63)$ & $27(15)$ & \\
\hline \multirow{2}{*}{$\begin{array}{l}\text { Hemorrhagic } \\
17(13.6)\end{array}$} & subarachnoid & $1(20)$ & $4(80)$ & $5(4)$ & \multirow[t]{2}{*}{0.87} \\
\hline & intracerebral & $2(17)$ & $10(83)$ & $12(9.6)$ & \\
\hline \multirow{3}{*}{$\begin{array}{l}\text { severity(NIHSS) } \\
125(100)\end{array}$} & Mild & $16(34)$ & $31(64)$ & $47(37.6)$ & \multirow[t]{3}{*}{0.01} \\
\hline & Moderate & $23(46)$ & $27(54)$ & $50(40)$ & \\
\hline & severe & $4(14)$ & $24(86)$ & $28(22.4)$ & \\
\hline \multirow{2}{*}{$\begin{array}{l}\text { Smoking } \\
42(33.6)\end{array}$} & Yes & $9(21)$ & $33(79)$ & $42(33.6)$ & \multirow[t]{2}{*}{0.03} \\
\hline & No & $34(41)$ & $49(59)$ & $83(66.4)$ & \\
\hline \multirow{2}{*}{$\begin{array}{l}\text { Risk factors } \\
104(83.2)\end{array}$} & yes & $38(36.5)$ & $66(63.5)$ & $104(83.2)$ & \multirow[t]{2}{*}{0.36} \\
\hline & No & $16(76)$ & $5(24)$ & $21(16.8)$ & \\
\hline \multirow{3}{*}{$\begin{array}{l}\text { Disability (mRS) } \\
\text { In discharge } \\
113(100)\end{array}$} & Mild & $16(41)$ & $23(59)$ & $39(34.5)$ & \multirow[t]{3}{*}{0.02} \\
\hline & Moderate & $18(44)$ & $23(56)$ & $41(36.2)$ & \\
\hline & Severe & $5(15)$ & $28(85)$ & $33(29.2)$ & \\
\hline \multirow{2}{*}{$\begin{array}{l}\text { Mortality } \\
12(9.6)\end{array}$} & No & $39(34.5)$ & $74(65.5)$ & 113(90.4) & \multirow[t]{2}{*}{0.93} \\
\hline & Yes & $4(33.3)$ & $8(66.7)$ & $12(9.6)$ & \\
\hline
\end{tabular}

${ }^{*}$ The serum sodium concentration $\left(\left[\mathrm{Na}^{+}\right]\right)<135 \mathrm{mmol} / \mathrm{L}$ (135 meq / L) considered as Hyponatremia and 135 to $145 \mathrm{mmol} / \mathrm{L}$ was defined as normal serum levels. Serum level assessment was performed with Latex particle agglutination test with Hitachi 912 auto analyzer in Ayatollah Rouhani Hospital Laboratory

${ }^{* *}$ chi-square or Fisher exact test were used between demographic and clinical variable with sodium serum levels

${ }_{* * *}$ Abbreviations: National Institute of Health Stroke Scale(NIHSS); Modified Rankin Scale (mRS)

Table 1: Stroke types, risk factors, severity, and early prognosis in Babol stroke patients with or without Hyponatremia

Of 17 patients (13.6\%) were hemorrhagic, 12 cases (9.6\%) ICH and 5 cases (4\%) were SAH. The difference in type of stroke with hyponatremia was statistically significant (IS 68(62\%) vs. HS 14(82\%), p=0.01).

The result of this study showed that 82 cases (65.6\%) of stroke survivors had hyponatremia in admission time. Of 85 thrombotic ischemic, 54 cases $(43.2 \%)$ had hyponatremia. while14 (11.2\%) of embolic stroke had hyponatremia ( $\mathrm{p}=0.81)$. However, this difference was not statistically significant. 
The frequency of different types of stroke and their demographic variables in patients with hyponatremia in comparison with normal sodium serum level stroke patients (Table 1). On one hand, History of smoking as a stroke risk factor was present in 42 stroke patients (33.6\%). Also, the difference between smoking or non-smoking in regard with hyponatremia was statistically significant $(\mathrm{p}=0.03)$.

The stroke risk factors such as HTN, IHD, DM and HLP were seen in 104 cases (83.2\%), in this patients66 cases (52.8\%) had hyponatremia, and 38 cases (30.4\%) of patients who had stroke risk factor were normal serum level of sodium ( $\mathrm{p}=0.36$ )

The Difference in admits score (NIHSS) in hyponatremia stroke patients vs. normal serum sodium level stroke patients was statistically significant $(\mathrm{p}=0.01)$. In other words, hyponatremia was more common in severe stroke patients. The difference in the degree of disability (mRS) of stroke patients in discharge time in stroke patients with hyponatremia versus stroke Patients with normal serum sodium was statistically significant $(\mathrm{p}=0.02)$. Increase in the degree of disability from admission time until discharge in stroke patients with hyponatremia versus stroke patients with normal serum sodium was statistically significant $(\mathrm{p}=0.02)$ (Table 1).

The mortality rate was 12 cases $(9.6 \%)$ of stroke patients after hospitalization. There was no statistically significant difference in mortality rate between the two groups $(p=0.93)$

\section{Discussion}

Frequency of hyponatremia in Babol stroke patients in this study was $66 \%$ which is high in comparison with other studies. In Soiza et al. study in Brazil, of 8540 stroke patient's hyponatremia prevalence was 14\% [10]. In the Rodrigues study et al. study 3585 patients with ischemic stroke, hyponatremia prevalence was 16\%. In Saleem et al. study in India, hyponatremia prevalence was 35\% [15]. The reasons for the great difference between low frequency (14-35\%) of hyponatremia in these studies and our study $(66 \%)$ could be due to different study methods, for example, due to few sample volumes. In addition, it can due to different lifestyles and the difference in health policy in risk factors control such as HTN, IHD and DM that can be a different result with current study with others. However, due to high frequency, it should be taken seriously and found reasons such as inappropriate Secretion of Antidiuretic Hormone (SIADH) or Cerebral Salt Wasting Syndrome (CSWS).

Based on their serum sodium level, in stroke patients, frequency of stroke risk factors such as HTN, IHD, DM. and HLP as a whole did not show a statistically significant difference in two groups, but smoking was more present in hyponatremia cases in comparison with normal sodium serum level of stroke patients. However, there is no definite data about the relationship between hyponatremia and the history of smoking as a risk factor in stroke patients, and if this relationship exists, its type and its significance are not determined. Given this finding, smoking as a risk factor should be taken for granted considering the relationship between the past histories of smoking in our hyponatremia stroke patients.

In relation to the association of stroke risk factors with hyponatremia, the Huang WY et al. study, DM was more prominent in hyponatremia stoke cases at admission time [16]. Perhaps some of these risk factors with hyponatremia are confirmed in future studies, these findings may be useful in the prevention and/or treatment of these patients.

Based on the findings of this study, the proportion of hyponatremia cases to normal serum sodium level cases was higher in patients with hemorrhagic stroke than ischemic patients. In Alam et al. study in Bangladesh, 23\% of HS and 21\% of IS patients had hyponatremia [17]. Also in some other studies such as Saleem et al. study, hyponatremia was more seen in hemorrhagic patients [14]. In Siddiqui's et al. study, hyponatremia was seen in $61 \%$ of HS cases versus $43 \%$ of IS cases study showed hyponatremia was seen more in hemorrhagic vs. ischemic strokes $[18,19]$. Though causes such as SIADH or CSWS which are more common in hemorrhagic stroke cases, can justify this difference, but there may be other causes for this difference.

Based on the findings of this study, the difference in severity of stroke in admission time instroke patients with hyponatremia versus normal group was statistically significant, with more severity in the cases of hyponatremia that was similar with Rodrigues et al. study(9). On other hand, in Badikillaya et al. study, no association was observed between stroke severity and hyponatremia [20]. Also, in Kembuan et al. study no association was detected between sodium blood disturbances and the severity of the acute stroke, although electrolyte disturbances were common in admission time [7]. If the relationship between hyponatremia and stroke severity is confirmed, the question arises whether hyponatremia leads to severe stroke or more severe stroke will lead to hyponatremia more.

Our findings also disclosed that hyponatremia is associated with the increased rate of disability in both admission and discharge time as well as an increased rate of disability from admission to discharge time. In Rodrigues et al. study, the grade of the disability in admission time increased until discharge accompanied by hyponatremia [9], which is in line with the results of this Study. How and through what mechanisms hyponatremia aggravates disability is subject to further scrutiny.

In current study, there was no statistically significant difference between mortality rate of stroke patients with hyponatremia and control groups, but in some studies mortality rate of stroke patients with electrolyte imbalance was higher than in patients with the normal level. In Kret et al. study, hyponatremia was associated with increased mortality within 1 month after the stroke [21]. In addition, in Soiza et al. study in Brazil, patients with hyponatremia in younger ages than 75 showed a significant difference in the 
prognosis of mortality [10]. In Huang et al. study, they concluded that hyponatremia was a predictor of mortality for three years in the first attack stroke and it can be a hallmark in that hyponatremia can be a strong prognostic factor for the acute phase [16]. In Rodrigues et al. study they found that hyponatremia had a statistically significant relationship with the mortality rate [9]. Despite these results, our findings did not confirm that hyponatremia was a strong predictor of increased mortality rate in the acute phase of hospitalized patients. In justifying the lack of relationship between hyponatremia and early mortality rate in our study, we can refer to the short time of hospitalization period prognosis (up to one week) in this study. The difference in the health care, medical and nursing services, and avoidance from excessive fluid therapy along with many other items was also involved in the rate of early mortality of these patients.

However, considering the findings of these studies and our study which disclosed the association of the increased rate of disability from admission to discharge time in hyponatremic cases. These patients should receive special care to prevent the rise of disabilities due to the fact that it is more likely to get worse in hyponatremic cases. This will finally lead to a decrease in cost and increase in community health. Since hyponatremia in strokes leads to a higher percentage of severe disabilities, it is suggested that new health policy and guidelines should be considered in stroke patients to maintain normal sodium serum level, and patients under treatment with lower sodium drugs should be monitored frequently for normal serum level of sodium.

The strength of our study is that it investigated the impact of hyponatremia on various aspects of stroke such as types, risk factors, prognosis, disability and mortality rate after stroke.

Limitation of this study was its small sample size, loss of control group, and short period follow-up time. Provide all the stroke risk factors together in the table (other than smoking) were another limitation.

\section{Conclusion}

Prevalence of hyponatremia is high in patients with acute stroke and can be considered as a predicting factor of stroke severity at admission and discharge time, with an increase in the degree of disability. The frequency of hyponatremia had no association with early mortality in the acute phase. It suggests a similar cohort study to conduct in multi-centers with larger sample size and long term follow-up.

\section{Acknowledgement}

This work was funded by BUMS, Iran (Proposal Number: 2791). We appreciate and thank of the personnel of Rouhani Hospital of Babol, especially Mrs. Nafiseh ezami for cooperation and assistance in this study.

\section{Ethics}

The study was approved by the ethics committee of Babol University of medical science (BUMS 940011-95.6.9).

\section{References}

1. Ahangar A, Khafri S, Saadat P, Alijanpour S (2019) The Role of Mineral Serum Level in Risk Factor of Stroke. Babol, North of Iran. J Neurol Exp Neurosci 5: 27-33.

2. Esenwa C, Gutierrez J (2015) Secondary stroke prevention: challenges and solutions. Vasc Health Risk Manag 11: 437-50.

3. Ahangar AA, Saadat P, Heidari B, Taheri ST, Alijanpour S (2018) Sex difference in types and distribution of risk factors in ischemic and hemorrhagic stroke. Int J Stroke 13: 83-6.

4. Hosseini AA, Sobhani-Rad D, Ghandehari K, Benamer HT (2010) Frequency and clinical patterns of stroke in Iran-Systematic and critical review. BMC Neurol 10: 72 .

5. Ahangar AA, Ashraf Vaghefi SB, Ramaezani M (2005) Epidemiological evaluation of stroke in Babol, northern Iran (2001-2003). Eur Neurol 54: 93-7.

6. Ahangar AA, Saravi M, Alijanpour S, Boora MM, Hoseinalipour S, et al. (2016) Comparison of Risk Factors of Stroke and Myocardial Infarction in Patients 15 to 45 Years in Affiliated Hospitals of Babol University of Medical Sciences. Zahedan J Res Med Sci 2016: e5332.

7. Kembuan M, Sekeon S (2014) Electrolyte disturbances among acute stroke patients in Manado, Indonesia. Global J Med Public Health 3.

8. Krummel T, Prinz E, Metten MA, Borni-Duval C, Bazin-Kara D, et al. Prognosis of patients with severe hyponatraemia is related not only to hyponatraemia but also to comorbidities and to medical management: results of an observational retrospective study. BMC Nephrol 17: 159.

9. Rodrigues B, Staff I, Fortunato G, McCullough LD (2014) Hyponatremia in the prognosis of acute ischemic stroke. J Stroke Cerebrovasc Dis 23: 850-4.

10. Soiza RL, Cumming K, Clark AB, Bettencourt-Silva JH, Metcalf AK, et al. Hyponatremia predicts mortality after stroke. Int J Stroke 10: 50-5.

11. Chawla A, Sterns RH, Nigwekar SU, Cappuccio JD (2011) Mortality and serum sodium: do patients die from or with hyponatremia? Clin J Am Soc Nephrol 6: $960-5$.

12. Sacco RL, Kasner SE, Broderick JP, Caplan LR, Culebras A, et al. (2013) An updated definition of stroke for the 21st century: a statement for healthcare professionals from the American Heart Association/American Stroke Association. Stroke 44: 2064-89.

13. Muchada M, Rubiera M, Rodriguez-Luna D, Pagola J, Flores A, et al. (2014) Baseline National Institutes of Health stroke scale-adjusted time window for intravenous tissue-type plasminogen activator in acute ischemic stroke. Stroke 45:1059-63.

14. Saleem S, Yousuf I, Gul A, Gupta S, Verma S (2014) Hyponatremia in stroke. Ann Indian Acad Neurol 17: 55-7.

15. Laville M, Burst V, Peri A, Verbalis JG. Hyponatremia secondary to the syndrome of inappropriate secretion of antidiuretic hormone (SIADH): therapeutic decision-making in real-life cases. Clin kidney J 6: i1-20. 
16. Huang WY, Weng WC, Peng TI, Chien YY, Wu CL, et al. (2012) Association of hyponatremia in acute stroke stage with three-year mortality in patients with first-ever ischemic stroke. Cerebrovasc Dis 34: 55-62.

17. Alam M, Uddin M, Rahman K, Ahmed S, Akhter M, et al. (2012) Electrolyte changes in stroke. Mymensingh Med J 21: 594-9.

18. Siddiqui MR, Islam QT, Haque MA, Iqbal MJ, Hossain A, et al. (2012) Electrolytes status in different type of acute stroke patients and their correlation with some common clinical presentation. J of Med 13:133-7.

19. Babaliche P, Madnani S, Kamat S (2017) Clinical profile of patients admitted with hyponatremia in the medical intensive care unit. Indian J Crit Care Med 21: 819-24.

20. Badikillaya VU, Tummi M, Pernenkil SR (2013) Hyponatraemia in head injuries caused by road traffic accidents. J Clin Diagn Res 7: 407-8.

21. Kret B, Lasek-Bal A, Holecki M, Hawrot-Kawecka A, Wilczyński K, et al. (2016) Prognostic relevance of hyponatremia after first-ever ischemic stroke. Ann Academiae Medicae Silesiensis 70: 127-32. 